\title{
Initial stage of interaction between gyrating relativistic electron beam and long- wavelength electromagnetic surface waves in cylindrical metallic waveguides partially filled with plasma
}

Cite as: Phys. Plasmas 26, 042118 (2019); https://doi.org/10.1063/1.5090238

Submitted: 25 January 2019 . Accepted: 03 April 2019 . Published Online: 22 April 2019

I. O. Girka (D), O. I. Girka (D), and M. Thumm (D)
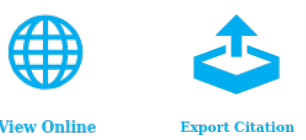

Where in the world is AIP Publishing? Find out where we are exhibiting next 


\title{
Initial stage of interaction between gyrating relativistic electron beam and long-wavelength electromagnetic surface waves in cylindrical metallic waveguides partially filled with plasma
}

Cite as: Phys. Plasmas 26, 042118 (2019); doi: 10.1063/1.5090238

Submitted: 25 January 2019 - Accepted: 3 April 2019 .

Published Online: 22 April 2019

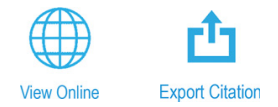

I. O. Girka, ${ }^{1, a)}$ (D) O. I. Girka, ${ }^{2}$ (D) and M. Thumm ${ }^{3}$ (iD

\author{
AFFILIATIONS \\ ${ }^{7}$ V.N. Karazin Kharkiv National University, Svobody sq., 4, 61022 Kharkiv, Ukraine \\ ${ }^{2}$ Plasma Technology Research Center, National Fusion Research Institute, 54004 Gunsan, South Korea \\ ${ }^{3}$ Karlsruhe Institute of Technology, IHM and IHE, Kaiserstr. 12, 76131 Karlsruhe, Germany

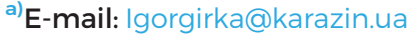

\begin{abstract}
Azimuthal surface waves are eigen electromagnetic waves of cylindrical metallic waveguides with plasma filling. Azimuthal surface waves of extraordinary polarization are known to be effectively excited by a beam of electrons gyrating in an axial external static magnetic field along large Larmor radii around the plasma column. Beam excitation of azimuthal surface waves was well studied theoretically in both linear and nonlinear approaches. An important limitation of the earlier studies was the approximation of zero axial wavenumber. In the present paper, perturbation theory is applied to account for finite magnitude of the axial wavenumber of electromagnetic surface waves in studying their excitation by an electron beam. The dependence of the wave growth rate on the axial wavenumber and parameters of the plasma waveguide is studied. The results obtained in this paper are of interest for application in plasma electronics.
\end{abstract}

Published under license by AIP Publishing. https://doi.org/10.1063/1.5090238

\section{INTRODUCTION}

Azimuthal surface waves (ASWs) are electromagnetic waves which propagate across the axis of cylindrical metallic waveguides both without any magnetic field and in the presence of an axial external static magnetic field $B_{0}{ }^{1}$ Absence of axial wavenumber provides the possibility to split the Maxwell equations for electromagnetic waves into two independent subsets. One of the latter describes ASWs with ordinary polarization (OASWs). They propagate with the components $E_{z}, H_{r}$, and $H_{\varphi}$ of the electromagnetic field, and their dispersion properties do not depend on the presence of the axial external static magnetic field $B_{0}$. The other subset of the Maxwell equations describes ASWs with extraordinary polarization (XASWs). They propagate with the components $H_{z}, E_{r}$, and $E_{\varphi}$ of the electromagnetic field, independently of the OASWs.

If the plasma particle density is sufficiently large and the $B_{0}$ is weak enough so that $\Omega_{e}{ }^{2}>\omega_{e}{ }^{2}$, XASWs propagate in two frequency ranges $^{1}$ (here $\Omega_{e}$ is the plasma (Langmuir) frequency of electrons, and $\omega_{e}$ is the electron cyclotron frequency). One of them, being referred to as low frequency (LF) range, includes the electron cyclotron frequency and is under consideration in this paper. The other range lies higherabove the upper hybrid frequency. It is out of the scope of the present study, although XASWs can be also excited there. ${ }^{2}$

The assumption that the Langmuir frequency is higher than the electron cyclotron frequency cannot be considered as a strong limitation of applicability of the results obtained in the present paper. For the conditions of a modest university laboratory, a plasma particle density of $n_{e} \sim 10^{12} \mathrm{~cm}^{-3}$ is not exotic. To provide the inequality $\Omega_{e}{ }^{2}$ $>\omega_{e}^{2}$ for this $n_{e}$, one should apply an external static magnetic field $B_{0}$ $\lesssim 3.2 \times 10^{-3} \sqrt{n_{e}} \sim 0.3 \mathrm{~T}$. Even stronger $B_{0}$ can be used if semiconductor plasmas are considered ${ }^{3}$ for which the density $n_{e} \sim 10^{19} \mathrm{~cm}^{-3}$ of $n$-type conductivity electrons is achievable.

In the opposite case, $\Omega_{e}^{2}<\omega_{e}^{2}$, the possibility of XASW excitation in the ion cyclotron frequency range by a gyrating beam of light ions in cylindrical metallic waveguides partially filled with plasma is demonstrated in Ref. 4.

The presence of the azimuthal component $E_{\varphi}$ of the wave electric field provides the effective interaction of XASWs with a flow of charged particles which gyrate in $B_{0}$ along large Larmor radii. ${ }^{4-}$ 
For the most beam-plasma parameters, LF ASWs with azimuthal wavenumber $m$ use to be excited due to interaction with the beam cyclotron harmonic $s=m$. This is true also for $m>1$. However, the neighboring harmonics $s=m \pm 1, m \pm 2$ can also contribute to the LF ASW excitation. Two physics mechanisms of resonance overlapping in the excitation of azimuthal surface waves by rotating relativistic electron beams were demonstrated in Ref. 7.

One of the most important drawbacks of all the previous numerous studies of XASW beam excitation consists in neglecting the possible nonzero axial wavenumber $k_{z}$ of the wave. For example, in large orbit gyrotrons, ${ }^{8,9}$ the electromagnetic cavity mode is close to cutoff (small $k_{z}$ ). However, account for $k_{z} \neq 0$ can be carried out with the help of the perturbation theory. This approach was demonstrated to be effective for studying the dispersion properties of long-wavelength electromagnetic surface waves with the arbitrary magnitude of the azimuthal wavenumber $m$ propagating along the plasma-metal boundary in an axial external static magnetic field ${ }^{10}$ and in a cylindrical waveguide partially filled by isotropic plasma. ${ }^{11}$ In the presence of nonzero $k_{z}$, the Maxwell equations do not split into independent subsets for OASWs and XASWs-these two modes appear to be coupled. However, for long-wavelength waves (with a small magnitude of the $k_{z}$ ), the coupling can be considered as weak and $k_{z}$ as the small parameter of the problem.

There is no doubt that numerical methods are powerful tools for solving the problems of plasma electrodynamics. For instance, the results of numerical study of the dispersion properties, wave field structure, and energy characteristics of traveling electromagnetic nonsymmetric surface waves with azimuthal wavenumbers $m= \pm 2, \pm 3$ in axial external static magnetic field were considered in Ref. 12. Influences of the effective collision frequency, axial current, dimensions of coaxial waveguide system, and plasma particle density radial profile on dispersion properties and attenuation of symmetric $(m=0)$ electromagnetic waves were numerically studied in Ref. 13 . The solution of the dispersion equation in the case of dipolar waves $(m= \pm 1)$ was reported to be a more complicated problem because of the presence of the set of eigen solutions of the dispersion relation.

This paper is organized as follows: application of the method of successive approximation to the description of the wave field spatial distribution is given in Sec. II. The method to properly describe the wave fields in the region where the electron beam gyrates is presented in Sec. III. The dispersion relation which describes the initial stage of the beam excitation of surface type electromagnetic waves with a positive azimuthal wavenumber and small axial wavenumber is derived in Sec. IV. The results of a comprehensive numerical analysis of the influence of the nonzero axial wavenumber on the wave growth rate are presented in Sec. V. The conclusions with discussions of the results are summarized in Sec. VI.

\section{DESCRIPTION OF THE WAVE FIELDS IN THE PLASMA REGION}

Electromagnetic waves propagating with axial wavenumber $k_{z}$, azimuthal wavenumber $m$, and angular frequency $\omega$ are under consideration. Their wave fields can be written as $E_{z}$ and $H_{z}=f(r) \exp \left[i\left(k_{z} z\right.\right.$ $+m \varphi-\omega t)]$. These waves are excited in a metallic cylindrical waveguide of radius $b$. The waveguide is partially filled by a cylindrical uniform plasma column of radius $a<b$ (see Fig. 1).
The electrodynamic properties of the plasma placed into a uniform axial external static magnetic field $\vec{B}_{0} \| \vec{z}$ are described by the permittivity tensor of a cold collisionless plasma. ${ }^{14}$ The expressions for the components of the permittivity tensor are as follows:

$$
\begin{gathered}
\varepsilon_{11}=\varepsilon_{22}=\varepsilon_{r}-\sum_{\alpha} \frac{\Omega_{\alpha}^{2}}{\omega^{2}-\omega_{\alpha}^{2}} \equiv \varepsilon_{1}, \\
\varepsilon_{12}=-\varepsilon_{21}=i \sum_{\alpha} \frac{-\omega_{\alpha} \Omega_{\alpha}^{2}}{\omega\left(\omega^{2}-\omega_{\alpha}^{2}\right)} \equiv i \varepsilon_{2}, \\
\varepsilon_{33}=\varepsilon_{r}-\sum_{\alpha} \frac{\Omega_{\alpha}^{2}}{\omega^{2}} \equiv \varepsilon_{3},
\end{gathered}
$$

where $\Omega_{\alpha}$ and $\omega_{\alpha}$ are the plasma and cyclotron frequencies of the particle species $\alpha$, respectively, and $\varepsilon_{r}$ is the dielectric constant of the metamaterial or of the crystal lattice of the semiconductor $\left(\varepsilon_{r}>1\right)$, for gas plasmas $\varepsilon_{r}=1$.

One can derive the following set of two second order differential equations for the axial components of the wave electric $E_{z}$ and magnetic $B_{z}$ fields from the Maxwell equations:

$$
\begin{gathered}
\frac{1}{r} \frac{\partial}{\partial r} \frac{r}{k_{x}^{2}} \frac{\partial B_{z}}{\partial r}-\left[1+\frac{m^{2}}{r^{2} k_{x}^{2}}-\frac{m}{r} \frac{\partial}{\partial r}\left(\frac{\mu}{k_{x}^{2}}\right)\right] B_{z}=\hat{K} E_{z}, \\
\frac{1}{r} \frac{\partial}{\partial r} r \frac{\partial E_{z}}{\partial r}-\left(k_{o}^{2}+\frac{m^{2}}{r^{2}}\right) E_{z}=\hat{M} B_{z} .
\end{gathered}
$$

The right hand sides of Eqs. (4) and (5) have the following form if small values of the first order in $k_{z}$ are taken into account:

$$
\hat{K} E_{z}=i N_{z}\left\{\frac{m}{r} E_{z} \frac{\partial}{\partial r}\left(\frac{1}{k_{x}^{2}}\right)+\frac{\mu k_{o}^{2}}{k_{x}^{2}} E_{z}+\frac{\partial}{\partial r}\left(\frac{\mu}{k_{x}^{2}}\right) \frac{\partial E_{z}}{\partial r}\right\},
$$

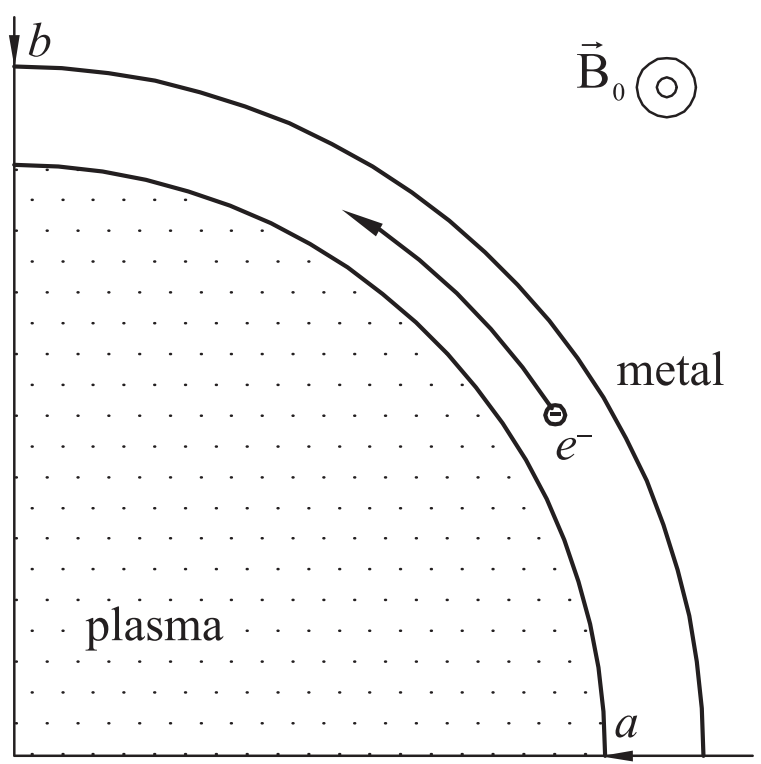

FIG. 1. Cross-section of the plasma-filled waveguide with a gyrating electron beam. 
$\hat{M} B_{z}=i N_{z}\left\{\frac{1}{N_{x}^{2}} \frac{\partial \mu}{\partial r} \frac{\partial B_{z}}{\partial r}+\left[\frac{\omega^{2}}{c^{2}} \mu+\frac{m}{r} \frac{\partial}{\partial r}\left(\frac{1}{N_{x}^{2}}\right)-\frac{\mu m}{r} \frac{\partial}{\partial r}\left(\frac{\mu}{N_{x}^{2}}\right)\right] B_{z}\right\}$.

The set of coupled equations (4) and (5) for the axial components of the electromagnetic field $E_{z}$ and $B_{z}$ for arbitrary values of the axial wavenumber $k_{z}$ and for a homogenous plasma in uniform axial static magnetic field was derived in Ref. 15.

In a plasma with the uniform particle density $n_{e}$, the depth of $H$ wave (ordinarily polarized) $k_{o}^{-1}$ and $E$-wave (extraordinarily polarized) $k_{x}^{-1}$ penetration into the plasma is determined through the components (1)-(3) of the permittivity tensor $\varepsilon_{i k}$

$$
\begin{aligned}
k_{o}^{2} & =-(\omega / c)^{2} \varepsilon_{3}+k_{z}^{2}, \quad k_{x}^{2}=(\omega / c)^{2} N_{x}^{2}, \\
N_{x}^{2} & =\left(\varepsilon_{1}-N_{z}^{2}\right)\left(\mu^{2}-1\right), \quad \mu=\varepsilon_{2} /\left(\varepsilon_{1}-N_{z}^{2}\right) .
\end{aligned}
$$

The condition that the real part of $N_{x}^{2}$ should be positive determines the frequency ranges within which the waves are of surface nature in the plasma, which means that the wave amplitudes decrease with going away from the plasma boundary into the plasma depth. The $\mathrm{LF}$ range is given by

$$
\left|\omega_{e}\right| \sqrt{\left(\Omega_{i}^{2}+c^{2} k_{z}^{2}\right) /\left(\Omega_{e}^{2}+\omega_{e}^{2}\right)}<\omega<\left|\omega_{e}\right|, \quad\left|\omega_{e}\right|<\omega<\omega_{1},
$$

where $\omega_{1}$ is the cut-off frequency for bulk modes

$$
\omega_{1} \approx-\frac{\left|\omega_{e}\right|}{2}+\sqrt{\frac{\omega_{e}^{2}}{4}+\frac{\Omega_{e}^{2}}{\varepsilon_{r}}+\frac{c^{2} \Omega_{e}^{2} k_{z}^{2}}{\left(\sqrt{\Omega_{e}^{2} \varepsilon_{r}+0.25 \varepsilon_{r}^{2} \omega_{e}^{2}}-0.5 \varepsilon_{r}\left|\omega_{e}\right|\right)^{2}}} .
$$

The range (9) coincides with that obtained for XASWs ${ }^{1}$ in the limiting case $k_{z} \rightarrow 0$. Note that $\operatorname{Re}\left(k_{o}^{2}\right)>0$ in the range (9) as well.

The solutions of Eqs. (4) and (5) can be found by the variation of constants

$$
\begin{gathered}
B_{z}=\left(A_{2}-A_{1} \int_{a}^{r} \frac{\tilde{\psi} k_{H}^{2} \hat{K} \varphi d r}{W(\psi, \tilde{\psi})}\right) \psi+A_{1} \tilde{\psi} \int_{0}^{r} \frac{\psi k_{H}^{2} \hat{K} \varphi d r}{W(\psi, \tilde{\psi})}, \\
E_{z}=\left(A_{1}-A_{2} \int_{a}^{r} \frac{\tilde{\varphi} \hat{M} \psi d r}{W(\varphi, \tilde{\varphi})}\right) \varphi+A_{2} \tilde{\varphi} \int_{0}^{r} \frac{\varphi \hat{M} \psi d r}{W(\varphi, \tilde{\varphi})} .
\end{gathered}
$$

In (11) and (12), the functions $\psi(r)$ and $\varphi(r)$ are solutions of Eqs. (4) and (5) with zero right hand sides, respectively, which are finite at the cylinder axis, $r=0$. The functions $\tilde{\psi}(r)$ and $\tilde{\varphi}(r)$ are the solutions of the same equations which are linearly independent of the functions $\psi$ and $\varphi$. The solutions (11) and (12) include two constants of integration $A_{1}$ and $A_{2}$ since the other two constants of integration are already chosen to satisfy the boundary conditions that the wave fields $E_{z}$ and $B_{z}$ should be finite at the waveguide axis, where the functions $\tilde{\varphi}$ and $\tilde{\psi}$ are singular. The Wronskian $W(\psi, \tilde{\psi})$ of the functions $\psi(r)$ and $\tilde{\psi}(r)$ is equal to

$$
W(\psi, \tilde{\psi})=\psi \frac{\partial \tilde{\psi}}{\partial r}-\tilde{\psi} \frac{\partial \psi}{\partial r} \propto \frac{k_{x}^{2}}{r} .
$$

The Wronskian of the functions $\varphi(r)$ and $\tilde{\varphi}(r)$ is inversely proportional to the radial coordinate $r$

$$
W(\varphi, \tilde{\varphi})=\varphi \frac{\partial \tilde{\varphi}}{\partial r}-\tilde{\varphi} \frac{\partial \varphi}{\partial r} \propto \frac{1}{r} .
$$

In the considered case of uniform plasma particle density and external static magnetic field, $\psi(r)=I_{m}\left(k_{x} r\right)$ is a modified Bessel function, $\tilde{\psi}(r)=K_{m}\left(k_{x} r\right)$ is a McDonald function, $\varphi(r)=I_{m}\left(k_{o} r\right)$, and $\tilde{\varphi}(r)=K_{m}\left(k_{o} r\right) .{ }^{16}$ Their Wronskians (13) and (14) are equal to each other

$$
W\left(I_{m}\left(k_{x} r\right), K_{m}\left(k_{x} r\right)\right)=W\left(I_{m}\left(k_{o} r\right), \quad K_{m}\left(k_{o} r\right)\right)=-1 / r .
$$

To derive the dispersion relation, one also needs the expression for the tangential (azimuthal) wave electric field $E_{\varphi}$

$$
E_{\varphi}(r)=\frac{i c}{\omega N_{x}^{2}} \frac{d B_{z}}{d r}+\frac{i \mu}{N_{x}^{2}} N_{\varphi} B_{z}+\frac{N_{z} N_{\varphi}}{N_{x}^{2}} E_{z}+\frac{c \mu N_{z}}{\omega N_{x}^{2}} \frac{d E_{z}}{d r} .
$$

Hereinafter, $N_{\varphi}=\mathrm{cm} /(\omega r)$.

\section{DESCRIPTION OF THE WAVE FIELDS IN THE BEAM REGION}

The electrodynamic properties of the space $a<r<b$, where the electron flow gyrates, are described by the permittivity tensor $\varepsilon_{i k}{ }^{(b)}$. 5,14 Its components, used in the following, are as follows:

$$
\begin{gathered}
\varepsilon_{11}^{(b)}=1+\frac{\Omega_{b}^{2}}{\omega \gamma} \sum_{s=-\infty}^{+\infty} s^{2}\left[\frac{\gamma\left(J_{s}^{2}(m)\right)^{\prime}}{(s-y) m\left|\omega_{e}\right|}+\frac{k J_{s}^{2}(m) r^{2}}{(s-y)^{2} c m^{2}}\right] \\
\varepsilon_{12}^{(b)}=\frac{i \Omega_{b}^{2}}{\omega\left|\omega_{e}\right|} \sum_{s=-\infty}^{+\infty} s\left[\frac{\left(J_{s}(m) J_{s}^{\prime}(m)\right)^{\prime}}{s-y}+\frac{J_{s}(m) J_{s}^{\prime}(m)}{(s-y) m}+\frac{J_{s}(m) J_{s}^{\prime}(m) k v r}{(s-y)^{2} c m}\right] \\
=-\varepsilon_{21}^{(b)}, \\
\varepsilon_{22}^{(b)}=1+\frac{\Omega_{b}^{2}}{\omega\left|\omega_{e}\right|} \sum_{s=-\infty}^{+\infty}\left[\frac{2\left(J_{s}^{\prime}(m)\right)^{2}}{s-y}+\frac{\left(J_{s}^{\prime}(m)\right)^{2} v^{2} y}{(s-y)^{2} c^{2}}+\frac{2 m J_{s}^{\prime}(m) J_{s}^{\prime \prime}(m)}{s-y}\right], \\
\varepsilon_{33}^{(b)}=1-\frac{\Omega_{b}^{2}}{\omega^{2} \gamma^{2}} .
\end{gathered}
$$

In (17)-(20), $\Omega_{b}^{2}=4 \pi e^{2} n_{b} m_{e}^{-1}$ is the plasma frequency of the electron flow squared, $y=\omega \gamma /\left|\omega_{e}\right|, \gamma$ is the relativistic Lorentz factor, $v$ is velocity of the electron flow, the superscript $(b)$ indicates that the value corresponds to the beam region $(a<r<b)$, and $J_{s}(x)$ a Bessel function of the first kind. A prime denotes the derivative with respect to the argument.

The electron flow particle density is assumed to be small, $\alpha \equiv n_{b} /$ $n_{e} \ll 1$. This results in violation of the inequality $\Omega_{e}{ }^{2}>\omega_{e}{ }^{2}$ assumed to be valid for the plasma region. In the beam region $(a<r<b)$, the plasma frequency is assumed to be sufficiently small, so that $\Omega_{b}{ }^{2}$ $<\omega_{e}{ }^{2}$, and the waves under study are of bulk nature. The electromagnetic wave fields are found from the Maxwell equations (4) and (5) with the following replacements: 


$$
\begin{aligned}
k_{o}^{2} & \rightarrow k_{o}^{(b) 2}=(\omega / c)^{2} \varepsilon_{33}^{(b)}-k_{z}^{2}, \quad k_{x}^{2} \rightarrow k_{x}^{(b) 2}=(\omega / c)^{2} N_{x}^{(b) 2} \\
N_{x}^{2} & \rightarrow N_{x}^{(b) 2}=\varepsilon_{22}^{(b)}-N_{z}^{2}+\left(\varepsilon_{12}^{(b)}\right)^{2}\left(\varepsilon_{11}^{(b)}-N_{z}^{2}\right)^{-1} \\
\mu & \rightarrow \mu^{(b)}=-i \varepsilon_{12}^{(b)} /\left(\varepsilon_{11}^{(b)}-N_{z}^{2}\right) .
\end{aligned}
$$

Smallness of the electron flow particle density makes it possible to neglect the right hand sides of Eqs. (4) and (5) and write down the solutions to the Maxwell equations in the following form:

$$
\begin{aligned}
& B_{z}^{(b)}=A_{3}\left[N_{m}^{\prime}\left(k_{x}^{(b)} b\right) J_{m}\left(k_{x}^{(b)} r\right)-J_{m}^{\prime}\left(k_{x}^{(b)} b\right) N_{m}\left(k_{x}^{(b)} r\right)\right], \\
& E_{z}^{(b)}=A_{4}\left[N_{m}\left(k_{o}^{(b)} b\right) J_{m}\left(k_{o}^{(b)} r\right)-J_{m}\left(k_{o}^{(b)} b\right) N_{m}\left(k_{o}^{(b)} r\right)\right] .
\end{aligned}
$$

The expressions (22) and (23) for both axial components of electromagnetic field $B_{z}^{(b)}$ and $E_{z}^{(b)}$ already satisfy the boundary conditions at the metallic wall $\left[E_{\varphi}{ }^{(b)}(b)=0\right.$ and $\left.E_{z}^{(b)}(b)=0\right]$

$$
E_{\varphi}^{(b)}(r)=\frac{i c}{\omega N_{x}^{(b) 2}} \frac{d B_{z}^{(b)}}{d r}+\frac{N_{z} N_{\varphi}}{N_{x}^{(b) 2}} E_{z}
$$

and that is why they contain only one constant of integration each $\left(A_{3}\right.$ and $A_{4}$ ).

\section{DISPERSION RELATION}

The four constants of integration $\left[A_{1}\right.$ and $A_{2}$ in (11) and (12), $A_{3}$ in (22), and $A_{4}$ in (23)] can be determined as the result of application of the following four boundary conditions. They are continuity of the tangential wave fields $E_{\varphi}, E_{z}, B_{\varphi}$, and $B_{z}$ at the plasma column interface $r=a$. To satisfy these boundary conditions, the determinant constructed from the coefficients nearby the constants $A_{i}$ should be equal to zero, which gives the dispersion relation. After calculating the determinant and neglecting the small terms of higher than the second order, the dispersion relation reads as

$$
D_{o} D_{x}=D_{1} .
$$

The general form of (25) is typical for dispersion relations of two modes with weak coupling. ${ }^{17}$

In (25), $D_{o}$ is dispersion relation of ordinary ASWs

$$
\begin{gathered}
D_{o}=a_{21} a_{33}-a_{31} a_{23}, \\
a_{21}=\frac{c k_{o}}{\omega} I_{m}^{\prime}\left(k_{o} a\right), \\
a_{23}=\frac{\omega}{c k_{o}^{(b)}}\left[J_{m}\left(k_{o}^{(b)} b\right) N_{m}^{\prime}\left(k_{o}^{(b)} a\right)-N_{m}\left(k_{o}^{(b)} b\right) J_{m}^{\prime}\left(k_{o}^{(b)} a\right)\right], \\
a_{31}=I_{m}\left(k_{o} a\right), \\
a_{33}=\left[J_{m}\left(k_{o} b\right) N_{m}\left(k_{o} a\right)-N_{m}\left(k_{o} b\right) J_{m}\left(k_{o} a\right)\right],
\end{gathered}
$$

and $D_{x}$ is dispersion relation of extraordinary ASWs

$$
\begin{gathered}
D_{x}=a_{12} a_{44}-a_{14} a_{42}, \\
a_{12}=\frac{1}{N_{x}} I_{m}^{\prime}\left(k_{x} a\right)+\frac{\mu N_{\varphi}}{N_{x}^{2}} I_{m}\left(k_{x} a\right), \\
a_{14}=\frac{\omega}{c k_{x}^{(b)}}\left[N_{m}^{\prime}\left(k_{x}^{(b)} b\right) J_{m}^{\prime}\left(k_{x}^{(b)} a\right)-N_{m}^{\prime}\left(k_{x}^{(b)} a\right) J_{m}^{\prime}\left(k_{x}^{(b)} b\right)\right],
\end{gathered}
$$

$$
\begin{gathered}
a_{42}=I_{m}\left(k_{x} a\right) \\
a_{44}=\left[N_{m}^{\prime}\left(k_{x}^{(b)} b\right) J_{m}\left(k_{x} a\right)-J_{m}^{\prime}\left(k_{x}^{(b)} b\right) N_{m}\left(k_{x} a\right)\right] .
\end{gathered}
$$

In (25), $D_{1}$ is their coupling parameter which has a value of the second order in the small parameter of the problem $k_{z}$

$$
\begin{aligned}
D_{1}= & a_{24} a_{42}\left(a_{13} a_{31}-a_{11} a_{33}\right)+\left(a_{14} a_{41}-a_{11} a_{44}\right)\left(a_{23} a_{32}-a_{33} a_{22}\right) \\
& +a_{13} a_{44}\left(a_{21} a_{32}-a_{31} a_{22}\right)+a_{41} a_{24} a_{12} a_{33} .
\end{aligned}
$$

Those elements in Eq. (36), which are given below, are linear in $k_{z}$

$$
\begin{gathered}
a_{11}=\frac{\mu k_{z} k_{o}}{k_{x}^{2}} I_{m}^{\prime}\left(k_{o} a\right)+\frac{m k_{z}}{a k_{x}^{2}} I_{m}\left(k_{o} a\right) \\
-i \int_{0}^{a} r I_{m}\left(k_{x} r\right) k_{x}^{2} \hat{K} I_{m}\left(k_{o} r\right) d r\left[\frac{1}{N_{x}} K_{m}^{\prime}\left(k_{x} a\right)+K_{m}\left(k_{x} a\right) \frac{\mu N_{\varphi}}{N_{x}^{2}}\right], \\
a_{13}=\frac{m k_{z}}{a k_{o}^{(b) 2}}\left[J_{m}\left(k_{o}^{(b)} a\right) N_{m}\left(k_{o}^{(b)} b\right)-J_{m}\left(k_{o}^{(b)} b\right) N_{m}\left(k_{o}^{(b)} a\right)\right], \\
a_{22}=-\frac{\mu k_{z}}{k_{x}} I_{m}^{\prime}\left(k_{x} a\right)-\frac{m k_{z}}{a k_{x}^{2}} I_{m}\left(k_{x} a\right) \\
\quad+i \frac{c k_{o}}{\omega} K_{m}^{\prime}\left(k_{o} a\right) \int_{0}^{a} r I_{m}\left(k_{o} r\right) \hat{M} I_{m}\left(k_{x} r\right) d r \\
a_{24}=\frac{m k_{z}}{a k_{x}^{(b) 2}}\left[N_{m}\left(k_{x}^{(b)} a\right) J_{m}^{\prime}\left(k_{x}^{(b)} b\right)-N_{m}^{\prime}\left(k_{x}^{(b)} b\right) J_{m}\left(k_{x}^{(b)} a\right)\right] \\
a_{32}=-i K_{m}\left(k_{o} a\right) \int_{0}^{a} r I_{m}\left(k_{o} r\right) \hat{M} I_{m}\left(k_{x} r\right) d r \\
a_{41}=i K_{m}\left(k_{o} r\right) \int_{0}^{r} r I_{m}\left(k_{x} r\right) k_{x}^{2} \hat{K} I_{m}\left(k_{o} r\right) d r .
\end{gathered}
$$

The dispersion relation (25) is analyzed here under the following resonance condition:

$$
\omega=\omega_{0}+\delta \omega=s\left|\omega_{e}\right| \gamma^{-1}+\delta \omega,
$$

where $s$ is an integer, $\omega_{0}$ is the wave eigenfrequency in the plasma waveguide without the electron beam, and $\delta \omega$ is the complex correction to the frequency caused by the interaction of the beam electrons with the plasma. The imaginary part of $\delta \omega$ is the wave growth rate.

\section{NUMERICAL ANALYSIS OF THE DISPERSION RELATION}

To carry out numerical analyses of the dispersion relation (25), the following dimensionless values are used in Figs. 2-5. The utilized abscissa axis is as it was in Refs. 1, 2, and 5: $k_{e f}=m c /\left(a \Omega_{e}\right)$. This is explained as follows. The characteristic azimuthal wavenumber of the waveguide with plasma column of the radius $a$ is determined by $k_{\varphi}$ $=m / a$ and the effective azimuthal wavenumber $k_{e f}$ is $m / a$ normalized by the skin-depth $\delta=c / \Omega_{e}$. Increasing $k_{e f}$ means decreasing plasma radius $a$ and/or decreasing plasma particle density $n_{e}$. The ordinate 


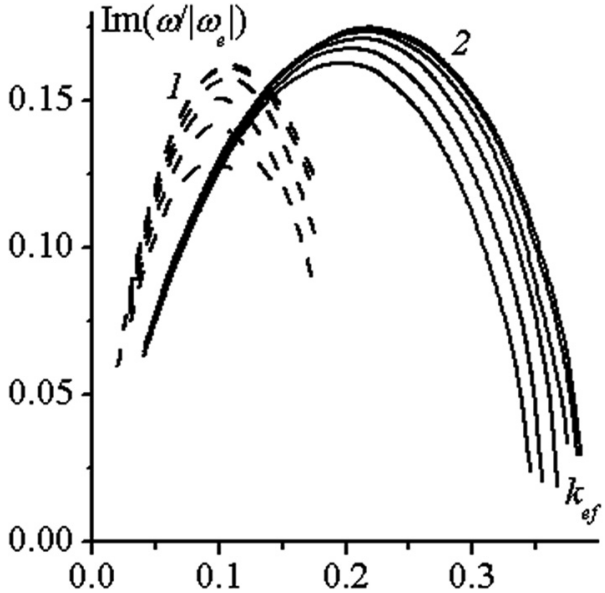

FIG. 2. Wave growth rate $\operatorname{Im}\left(\omega /\left|\omega_{e}\right|\right)$ vs $k_{e f}$ and $k_{z} a$ for $Z=7.5, \alpha=0.01, b /$ $a=1.2$, and $m=1,2$.

axis is chosen as the imaginary part of the frequency normalized by the electron cyclotron frequency, $\operatorname{Im}\left(\omega /\left|\omega_{e}\right|\right)$, except in Fig. 3 in which the influence of the axial external static magnetic field $B_{0}$ is examined. Normalization by $\left|\omega_{e}\right|$ is improper for studying the effect of $B_{0}$ on excitation of long wavelength electromagnetic waves since $\left|\omega_{e}\right|$ is proportional to $B_{0}$. That is why in Fig. 3 the imaginary part of the frequency normalized by the plasma frequency, $\operatorname{Im}\left(\omega / \Omega_{e}\right)$, is chosen as ordinate.

All Figs. 2-5 exhibit two sets of curves. One of these sets presents the wave growth rate $\operatorname{Im}(\omega)$ for $Z \equiv \Omega_{e} /\left|\omega_{e}\right|=7.5, \alpha=0.01, b / a=1.2$, and $m=1$ (shown by dashed curves) which is chosen to serve for comparison. The other set contains six curves and depicts the reduction of the wave growth rate with increasing axial wavenumber from $k_{z} a=0$ to $k_{z} a=0.2,0.4,0.6,0.8,1$. Within this range of the dimensionless variable $k_{z} a$, the condition of applicability of the method of successive approximations is valid. For the case of long wavelength

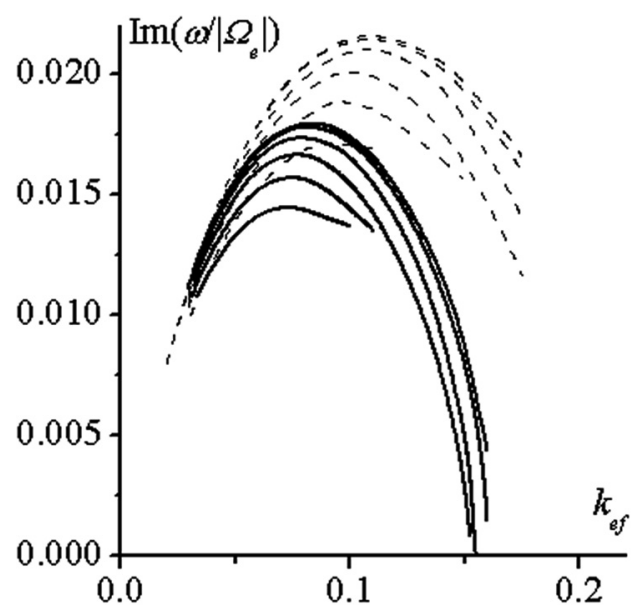

FIG. 3. Wave growth rate $\operatorname{Im}\left(\omega /\left|\omega_{e}\right|\right)$ vs $k_{e f}$ and $k_{z} a$ for $Z=10$ (solid curves) and $Z=7.5$ (dashed curves), $\alpha=0.01, b / a=1.2$, and $m=1$.

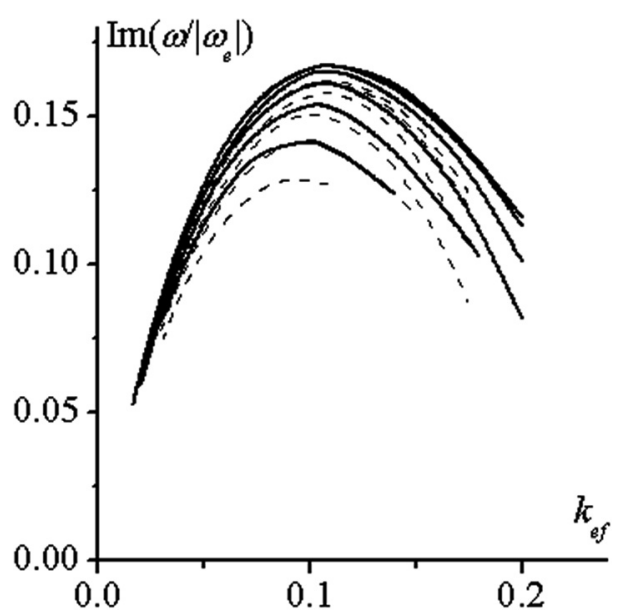

FIG. 4. Wave growth rate $\operatorname{Im}\left(\omega /\left|\omega_{e}\right|\right)$ vs $k_{\text {ef }}$ and $k_{z} a$ for $Z=7.5, \alpha=0.01, b /$ $a=1.1$ (solid curves), and $b / a=1.2$ (dashed curves), and $m=1$.

surface wave propagation in a cylindrical metallic waveguide entirely filled by plasma, this condition was derived in Ref. 11 analytically as

$$
\left(k_{z} a\right)^{2} \ll 8 m^{2}\left(1+k_{e f}^{2}\right) .
$$

During carrying out the calculations in the present paper, specific attention is given to follow the change of the real part $\left[\operatorname{Re}\left(\omega /\left|\omega_{e}\right|\right)\right]$ of the wave frequency. In the following, the maximum deviation of $\operatorname{Re}\left(\omega /\left|\omega_{e}\right|\right)$ calculated for $k_{z} a=1.0$ from that calculated for $k_{z} a=0$ is observed for the set of beam-plasma parameters which correspond to the conditions of Fig. 5. In this case, $\operatorname{Re}\left(\omega /\left|\omega_{e}\right|\right)$ increases by $27 \%$ from $\operatorname{Re}\left(\omega /\left|\omega_{e}\right|\right)=0.623$ to 0.793 with increasing $k_{z} a$ from $k_{z} a=0$ to $k_{z} a=1.0$.

Increase of $k_{z}$ from $k_{z} a=0$ to $k_{z} a=0.2$ causes a negligible decrease in $\operatorname{Im}\left(\omega /\left|\omega_{e}\right|\right)$, and the curves which correspond to $k_{z} a=0$ and $k_{z} a=0.2$ are almost indistinguishable in Figs. 2 and 4.

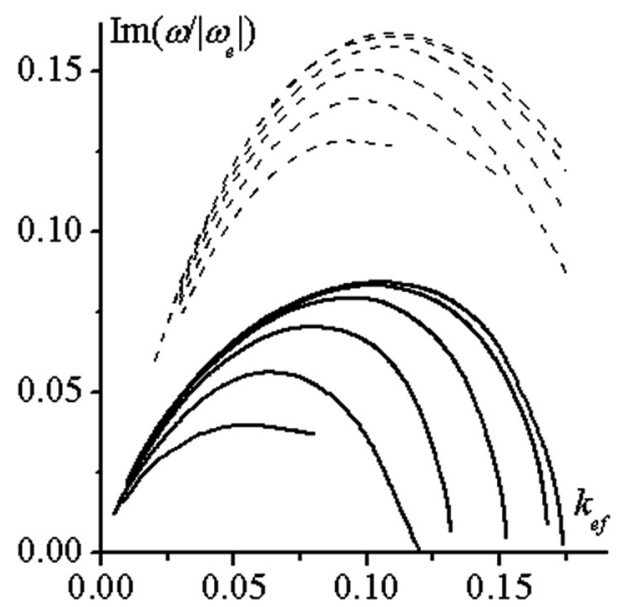

FIG. 5. Wave growth rate $\operatorname{Im}\left(\omega /\left|\omega_{e}\right|\right)$ vs $k_{\text {ef }}$ and $k_{z}$ a for $Z=7.5, \alpha=0.001$ (solid curves) and $\alpha=0.01$ (dashed curves), $b / a=1.2$, and $m=1$. 
In Fig. 2, the wave growth rates are calculated for the following beam-plasma parameters: $Z=\Omega_{e} /\left|\omega_{e}\right|=7.5, \alpha=0.01$, and $b / a=1.2$. The two sets of curves correspond to two different magnitudes of the azimuthal wavenumber: $m==1$ (dashed curves) and $m=2$ (solid lines). The $m$-values are displayed nearby the sets. Increase of the axial wavenumber from $k_{z}=0$ to $k_{z} a=1.0$ for the wave with $m=1$ is accompanied by a $20 \%$ decrease in the maximum growth rate from $\operatorname{Im}\left(\omega /\left|\omega_{e}\right|\right)=0.162$ which is observed for $k_{e f}=0.105$ to $\operatorname{Im}\left(\omega /\left|\omega_{e}\right|\right)$ $=0.129$ which is achieved for smaller $k_{e f}=0.09$. In other words, the magnitude of $k_{\text {ef }}$ for which the growth rate reaches its maximum decreases by $14 \%$. This tendency, decreasing of $k_{e f}$ value, for which the growth rate reaches the maximum magnitude, with increasing product $k_{z} a$, is a common feature of the curves in all Figs. 2-5.

To demonstrate how the increase in the azimuthal wavenumber $m$ influences the dependence of the growth rate on the axial wavenumber, the growth rates are calculated also in Fig. 2 for the same beamwaveguide parameters as for the wave with $m=1$ except $m$ which is increased to $m=2$. In this case, the maximum growth rate decreases by $7 \%$ from $\operatorname{Im}\left(\omega /\left|\omega_{e}\right|\right)=0.175$ which is observed for $k_{e f}=0.22$ when $k_{z}=0$ to $\operatorname{Im}\left(\omega /\left|\omega_{e}\right|\right)=0.163$ which is achieved for $k_{e f}=0.2$ when $k_{z} a=1.0$. The position of the growth rate maximum shifts only by $9 \%$. This result (weaker effect of axial wavenumber on the beam wave excitation for larger azimuthal wavenumbers) is predictable from the point of view of general physics since the wavenumber ratio $k_{z} / k_{\varphi}$ is smaller for larger $m$.

To show the effect of the external static axial magnetic field $B_{0}$ on the beam wave excitation, the growth rates are calculated in Fig. 3 for weaker $B_{0}$. All the beam-plasma waveguide parameters are the same as in Fig. 2 except $Z$, which is increased to $Z=10.0$. This results in a decrease in $B_{0}$ by $25 \%$. In this case, the maximum growth rate decreases by $19 \%$ from $\operatorname{Im}\left(\omega /\left|\omega_{e}\right|\right)=0.18$, which is observed for $k_{e f}$ $=0.08$ when $k_{z}=0$, to $\operatorname{Im}\left(\omega /\left|\omega_{e}\right|\right)=0.145$ which is achieved for $k_{e f}$ $=0.07$ when $k_{z} a=1.0$. So the position of the growth rate maximum shifts only by $12 \%$. The decrease in $B_{0}$ is accompanied by a weaker influence of the axial wavenumber on the wave growth rate.

The influence of the relative gap width $(b-a) / a$ on the dependence of the growth rate on axial wavenumber is shown in Fig. 4. To calculate the growth rates in Fig. 4, the same beam-plasma parameters are used as in Fig. 2 except the gap width which is smaller, $(b-a) / a$ $=0.1$ (solid curves), in this case. The maximum wave growth rate decreases by $15 \%$ from $\operatorname{Im}\left(\omega /\left|\omega_{e}\right|\right)=0.167$ in Fig. 4, which is observed for $k_{e f}=0.105$ when $k_{z}=0$, to $\operatorname{Im}\left(\omega /\left|\omega_{e}\right|\right)=0.142$ which is achieved for $k_{\text {ef }}=0.1$ when $k_{z} a=1.0$. So the position of the growth rate maximum shifts only by $5 \%$. The decrease in the gap width is also accompanied by a weaker influence of the axial wavenumber on the wave growth rate.

The wave beam-plasma instability growth rate is well-known to decrease with decreasing beam particle density. To demonstrate the effect of the parameter $\alpha$ on the dependence of the $\operatorname{Im}\left(\omega /\left|\omega_{e}\right|\right)$ versus the axial wavenumber, the growth rates are calculated in Fig. 5 for the same beam-plasma waveguide parameters as in Fig. 2 except $\alpha$ which is decreased by ten times to $\alpha=0.001$ (solid curves). It should be noted that the classical theory of the beam-plasma instability ${ }^{18}$ predicts the decrease in the wave maximum growth rate caused by a decrease in the beam particle density by ten times by the factor $\approx 0.464$, whereas the numerical analysis gives 0.525 which can be considered as a good qualitative agreement. The growth rate reaches its maximum for $k_{z}=0$ in Fig. 5 for the same magnitude of $k_{e f}=0.105$ as in Fig. 2. The maximum wave growth rate decreases by $53 \%$ from $\operatorname{Im}\left(\omega /\left|\omega_{e}\right|\right)$ $=0.085$ in Fig. 5 which is observed when $k_{z}=0$ to $\operatorname{Im}\left(\omega /\left|\omega_{e}\right|\right)=0.04$ which is achieved for $k_{e f}=0.05$ when $k_{z} a=1.0$. This means that the position of the growth rate maximum shifts by $52 \%$. The decrease in the beam particle density enhances the influence of the axial wavenumber on the wave growth rate.

As a practical example to evaluate the possibility of experimental realization of long wavelength surface wave excitation, the following parameters of the beam-plasma system are chosen. ${ }^{19}$ The plasma frequency is $\Omega_{e} \approx 1.261 \times 10^{11} \mathrm{~s}^{-1}$ for the plasma particle density $n_{e}$ $\approx 5 \times 10^{12} \mathrm{~cm}^{-3}$. The effective wavenumber is $k_{e f} \approx 0.059$ for the first azimuthal wavenumber $m=1$ and the plasma column radius $a$ $\approx 4 \mathrm{~cm}$. The electron cyclotron frequency is $\left|\omega_{e}\right| \approx 1.616 \times 10^{10} \mathrm{~s}^{-1}$ for the external static axial magnetic field $B_{0} \approx 920 \mathrm{G}$. The wave radial phase shift $\Delta \varphi$ within the gap $a<r<b \approx 4.8 \mathrm{~cm}$, where the electron flow gyrates, appears to be small, $\Delta \varphi \approx 0.167$. The beam electrons move with the velocity $v \approx 0.923 c$, which corresponds to the relativistic Lorentz factor $\gamma \approx 2.591$. The long wavelength surface waves with $k_{z}=0.09 \mathrm{~cm}^{-1}$ are excited with the frequency $f \approx 1 \mathrm{GHz}$ and the growth rate $\operatorname{Im}(\omega) \approx 7.8 \times 10^{7} \mathrm{~s}^{-1}$.

\section{CONCLUSIONS}

In the present paper, the initial stage of surface type electromagnetic wave excitation by a relativistic electron flow which gyrates in a narrow gap separating a dense plasma column from a metallic wall of a cylindrical waveguide is studied. The consideration is limited to the case of long-wavelength waves with small magnitudes of axial wavenumber $k_{z}$; however, the azimuthal wavenumber $m$ can have an arbitrary magnitude. The assumption of small axial wavenumber makes it possible to apply the theory of successive approximations with account for the small values of the first order in $k_{z}$ in the expressions for the electromagnetic wave fields. This results in deriving the dispersion relation (25) which has the form typical for coupled waves. In the present case, these coupled waves are ordinarily and extraordinarily polarized electromagnetic waves, which are known to propagate independently in the limit $k_{z} \rightarrow 0$, in which they are surface flute waves. ${ }^{1}$ They are weakly coupled by the small axial wavenumber $k_{z}$.

Applicability of the theory of successive approximations for studying the long-wavelength wave beam excitation is demonstrated. The dispersion relation, which describes the initial (linear in respect to the wave amplitude) stage of the wave excitation, is derived. The excitation is caused by efficient beam-wave interaction. The model of the electron beam as a flow of oscillators is applied. ${ }^{14}$ The wave growth rate which is the positive imaginary part of the complex frequency arises due to existence of resonant denominators in the expressions (17)-(19) for the permittivity tensor. These resonances correspond to the harmonics of electron cyclotron frequency.

The dispersion relation is analyzed numerically. The influence of the beam-plasma waveguide parameters on the wave growth rate is studied. In general, increasing axial wavenumber results in decreasing the maximum growth rate and increasing the plasma particle density and plasma column radius for which the waves are excited most efficiently. The influence of the axial wavenumber on the wave growth rate increases with decreasing beam particle density and azimuthal wavenumber, and decreases with decreasing the external static axial magnetic field and gap width within which the electron flow gyrates. 
The results obtained in this paper are of interest for plasma electronics, in particular, for solid state electronics. ${ }^{3}$

\section{ACKNOWLEDGMENTS}

This work was supported by the RD Program of Plasma Convergence \& Fundamental Research through the National Fusion Research Institute of Korea (NFRI) funded by the Government funds and Ministry of Education and Science of Ukraine research Grant No. 0119 U002526.

\section{REFERENCES}

${ }^{7}$ V. Girka, I. Girka, and M. Thumm, Surface Flute Waves in Plasmas: Theory and Applications (Springer-Verlag, Cham/Heidelberg/New York/Dordrecht/ London, 2014).

${ }^{2}$ V. O. Girka, I. O. Girka, and I. V. Pavlenko, "Excitation of azimuthal surface modes by relativistic flows of electrons in high-frequency range," Plasma Phys. Rep. 37(5), 447-454 (2011).

${ }^{3}$ C.-T. Sah, Fundamentals of Solid State Electronics (World Scientific Publishing, 1991).

${ }^{4}$ V. O. Girka, I. O. Girka, and I. V. Pavlenko, "Excitation of ion azimuthal surface modes in a magnetized plasma by annular flow of light ions," Prog. Electromagn. Res. M (PIERM) 21, 267-278 (2011).

${ }^{5}$ V. O. Girka, I. O. Girka, Y. I. Morgal, and I. V. Pavlenko, "Excitation of azimuthal surface modes by annular electron beams in the range of electron cyclotron frequency," Phys. Scr. 84, 025505 (2011).

${ }^{6}$ V. O. Girka and S. Yu. Puzyr'kov, "Nonlinear interaction of an annular electron beam with azimuthal surface waves," Plasma Phys. Rep. 28(4), 351-358 (2002).

${ }^{7}$ I. O. Girka, I. V. Pavlenko, and M. Thumm, "Two mechanisms of resonance overlapping in excitation of azimuthal surface waves by rotating relativistic electron beams," Phys. Plasmas 25(5), 052111 (2018).

${ }^{8}$ V. L. Bratman, Yu. K. Kalynov, V. N. Manuilov, and S. V. Samsonov, "Large orbit gyrotron at submillimeter waves," in Proceedings of the Conference on Digest 30th International Conference on Infrared and Millimeter Waves and 13th International Conference on Terahertz Electronics, Williamsburg, VA, USA (2005), pp. 443-444.

${ }^{9}$ V. L. Bratman, Yu. K. Kalynov, V. N. Manuilov, and S. V. Samsonov, "Largeorbit gyrotron operation at submillimeter waves," in Proceedings of the 6th International Workshop on Strong Microwaves in Plasmas, Nizhny Novgorod, 2005, edited by A. G. Litvak (Institute of Applied Physics, Russian Academy of Sciences, Nizhny Novgorod, 2006), Vol. 1, pp. 150-155.

${ }^{10} \mathrm{~V}$. O. Girka and I. O. Girka, "Asymmetric long-wavelength surface modes of isotropic plasma waveguides,” Plasma Phys. Rep. 28(8), 682-689 (2002).

${ }^{11}$ V. O. Girka and I. O. Girka, "Asymmetric long-wavelength surface modes in magnetized plasma waveguides entirely filled with plasma," Plasma Phys. Rep. 28(11), 916-924 (2002).

${ }^{12}$ N. A. Azarenkov, V. P. Olefir, and A. E. Sporov, "Quadrupole and octopole electromagnetic waves in slightly nonuniform magnetized plasma column," Phys. Scr. 63, 36-42 (2001).

${ }^{13}$ V. P. Olefir and A. E. Sporov, "Symmetric and dipolar electromagnetic waves in coaxial structure filled by non-uniform dissipative plasma with azimuth magnetic field," Prob. At. Sci. Technol., No. 1, Ser.: Plasma Phys. (17), 47-49 (2011).

${ }^{14}$ A. F. Alexandrov, L. S. Bogdankevich, and A. A. Rukhadze, Principles of Plasma Electrodynamics (Springer Verlag, Heidelberg, 1984).

${ }^{15}$ B. A. Aronov, L. S. Bogdankevich, and A. A. Rukhadze, "Electromagnetic irradiation under excitation of surface waves in plasma by electron beam," Tech. Phys. 43(12), 2493-2498 (1973) (in Russian).

${ }^{16} \mathrm{M}$. Abramowitz and I. Stegun, in Handbook of Mathematical Functions with Formulas, Graphs, and Mathematical Tables, Applied Mathematics Series (National Bureau of Standards, Washington, 1972).

${ }^{17}$ S. E. Miller, "Coupled wave theory and waveguide applications," Bell Syst. Tech. J. 33(3), 661-719 (1954).

${ }^{18}$ A. I. Akhiezer and Ya. B. Fainberg, "On the interaction of a charged particle beam with electron plasma,” Dokl. Akad. Nauk SSSR 69, 555-556 (1949) (in Russian) [Ukr. J. Phys. 53, 87-88 (2008), available at http://ujp.bitp.kiev.ua/ index.php?item $=\mathrm{j} \& \mathrm{id}=110$.

${ }^{19}$ Yu. E. Kolyada, Ya. B. Fainberg, E. A. Kornilov, V. V. Ognivenko, and V. A. Kiyashko, "Interaction of a high-current electron beam with a plasma in open cavity in a mirror system," Sov. Tech. Phys. Lett. 2, 348-350 (1976). 